\title{
Design a Membrane System for Matrix Multiplication
}

\author{
Lian Ye $\mathrm{e}^{\mathrm{a}, \mathrm{b}}$, Ping Guo ${ }^{\mathrm{a}, \mathrm{b}}$ \\ ${ }^{a}$ College of Computer Science, Chongqing University, Chongqing 400030, China \\ ${ }^{b}$ Chongqing Key Laboratory of Software Theory and Technology, Chongqing 400044, China
}

\begin{abstract}
Membrane systems are a framework of distributed parallel computing model$\mathrm{s}$, inspired by some basic features of biological membranes. In this paper, a membrane computing system named $\Pi_{\mathrm{MM}}$ is proposed to solve multiplication operation with matrices by its parallelism. It mainly includes three stages: firstly, construct new embedded membranes and input the values of two matrices; secondly, divide the membranes to generate new membranes according to the rows of matrix; finally, the solutions are obtained in each row membranes under the relatively calculation rules side by side. An instance is given to show the whole procedure how to solve the matrix multiplication by the $\Pi_{\mathrm{MM}}$.

Keywords: Cell-like P systems, Matrix multiplication, Parallel computing, Membrane Computing
\end{abstract}

\section{Introduction}

Matrix operations have been widely used in the field of data mining, such as image processing, text mining, recommendation system, bioinformatics and so on. Matrix multiplication is one of important matrix arithmetic operations.

5 Membrane Computing is an emerging branch within Natural Computing[1], this new model of computation starts from the assumption that the processes taking place in the compartmental structure of a living cell can be interpret-

\footnotetext{
${ }^{*}$ Corresponding author

Email addresses: ylredleaf@cqu.edu.cn (Lian Ye), guoping@cqu.edu.cn (Ping Guo)
}

Preprint submitted to Journal of ${ }^{A} T_{E} X$ Templates

April 19, 2016

(C) 2016. This manuscript version is made available under the Elsevier user license http://www.elsevier.com/open-access/userlicense/1.0/ 
ed a computations. Membrane system is the computing system based on the principle of Membrane Computing, which consists of a hierarchical arrangement of membranes embedded in a skin membrane, and delimiting regions or compartments where multi-sets of objects and sets of evolution rules are placed. $\mathrm{P}$ systems are not used as a computing paradigm, but rather as formalism for describing the behavior of the system to be modeled.

On the one hand, it is commonly accepted the belief that living systems perform some information processing to keep them far from dynamical equilibrium as well as to accomplish several particular tasks[2] allowing them to react and to adapt to the environment they live in.

On the other hand, people look at the way nature computes with the purpose to abstract its information processing mechanisms in such a way to obtain new computational paradigms that can be put beside traditional ones to tackle specific problems, like NP problems in a feasible time. SAT problem was solved by the splitting rule of membrane system[3], and the HPP problem was also solved by the generating rule[4], A hybrid membrane evolutionary algorithm was proposed for solving constrained optimization problems[5].

In this paper, we propose a $\mathrm{P}$ system named $\Pi_{\mathrm{MM}}$ to solve matrix arithmetic, which is a new field in membrane computing. Of course, the new computing system is based on our previous works. We designed single-membrane arithmetic $\mathrm{P}$ system without priority rules to simplify the structure of the system greatly and improve the efficiency of computations at first[6]. Then we have designed an arithmetic expression evaluations system to provide an effective method for applications of numerical computation with membrane computing[7], and proposed different membranes for implementing primary Boolean and relational operations respectively for evaluating a logical expression[8].In Ref.[9], a fraction arithmetic $\mathrm{P}$ system was presented for performing addition, subtraction, 35 multiplication and division on fractions through designing the rules with priority, and then a method of fraction reduction was proposed[10]. The fraction arithmetic $\mathrm{P}$ system is considered as a calculator component in $\Pi_{\mathrm{MM}}$, once the former operands are sending into the component, the operands of arithmetic 
result will be given out.

The organization of the paper is as follows: the foundation of $\mathrm{P}$ systems and the standard notations used for matrices and definition of multiplication operation with matrices are introduced in section 2 ; the details of $\Pi_{M M}$, including the membrane structure and evolving rules are proposed in section 3. In section 4 , an example is presented to show how the $\Pi_{\mathrm{MM}}$ works. The conclusions are 45 proposed in the final section.

\section{Preliminaries}

In membrane computing there are basically three ways to consider computational devices: cell-like systems[11, 12], tissue-like systems[13] and Neural-1ike Systems[14, 15]. The first one, using the biological membranes arranged hierarchically, inspired from the structure of the cell; the second one using the biological membranes placed in the nodes of a graph, inspired from the cell inter-communications in tissues; the third one, Spiking neural P systems are a class of distributed parallel computing devices inspired from the way neurons communicate by means of spikes. Our work is focused on the cell-like systems.

55 So all the basic knowledge about cell-like systems and the description of matrix operation are given in this section.

\subsection{Cell-like P systems}

The main syntactic ingredients of a cell-like $\mathrm{P}$ system are the membrane structure, the multi-sets, and the evolution rules. A membrane structure consists of several membranes arranged in a hierarchical structure inside a main membrane (the skin), and delimiting regions (the space in-between a membrane and the immediately inner membranes). Each membrane identifies a region inside the system. Regions defined by a membrane structure contain objects corresponding to chemical substances present in the compartments of a cell.

65 The objects can be described by symbols or by strings of symbols, in such a way that multi-sets of objects are placed in regions of the membrane structure. 
The objects can evolve according to given evolution rules, associated with the regions. The semantics of the cell-like membrane systems is defined through a nondeterministic and synchronous model, by introducing the concepts of configuration, transition step, and computation.

A basic transition $\mathrm{P}$ system of degree $m \geq 1$ is a tuple[1],

$$
\Pi=\left(O, \mu, \omega_{1}, \cdots, \omega_{m},\left(R_{1}, \rho_{1}\right),\left(R_{2}, \rho_{2}\right), \cdots,\left(R_{m}, \rho_{m}\right), i_{o}\right)
$$

where,

(i) $O$ is the alphabet of the system;

(ii) $\mu$ is a membrane structure consisting of $\mathrm{m}$ membranes, which are labeled 75 by numbers in the set $\{1, \cdots, \mathrm{n}\}$;

(iii) $\omega_{1} \cdots \omega_{m}$ are multi-sets, representing the objects initially present in the regions $(1, \cdots, \mathrm{m})$ of the system;

(iv) $R_{1}, \cdots, R_{m}$ are finite sets of evolution rules associated with the regions $(1, \cdots, \mathrm{m})$ of $\mu ;\left(\rho_{1}, \cdots, \rho_{m}\right)$ are strict partial order relations defined over

so $\left(\mathrm{R}_{1}, \cdots, \mathrm{R}_{m}\right)$ respectively, specifying a priority relation among the evolution rules; The rule can be described as the form $\left(u \rightarrow v, \rho_{i}\right)$, where, $u \rightarrow v$ is rewrite rule, and $\rho_{i}(1 \leq i \leq m)$ indicate the priority.

$(\mathrm{v}) i_{o}$ is indicating the output region.

\subsection{Matrix Multiplication}

More generally, it is possible multiply a matrix A times a matrix B if the number of columns of A equals the number of rows of B. The first column of the product is determined by the first column of B; that is, the first column of $\mathrm{AB}$ is $\mathrm{Ab} \mathrm{b}_{1}$, the second column of $\mathrm{AB}$ is $\mathrm{Ab} \mathrm{b}_{2}$, and so on. Thus the product $\mathrm{AB}$ is the matrix whose columns are $\mathrm{Ab}_{1}, \mathrm{Ab}_{2}, \cdots, \mathrm{Ab}_{n}$ :

$$
\mathrm{A} B=\left(A b_{1}, A b_{2}, \cdots, A b_{n}\right)
$$

The $(i, j)$ entry of $\mathrm{AB}$ is the $i_{t h}$ entry of the column vector $\mathrm{Ab}_{j}$. It is determined by multiplying the $i_{t h}$ row vector of A times the $j_{t h}$ column vector of B. 
Definition 1. if $\mathrm{A}=\left(\mathrm{a}_{i j}\right)$ is a $(\mathrm{m} \times \mathrm{k})$ matrix and $\mathrm{B}=\left(\mathrm{b}_{i j}\right)$ is a $(\mathrm{k} \times \mathrm{n})$ matrix, then the product $\mathrm{AB}=\mathrm{C}=\left(\mathrm{c}_{i j}\right)$ is the $(\mathrm{m} \times \mathrm{n})$ matrix whose entries are defined by

$$
C_{i j}=a_{i} b_{j}=\sum_{l=1}^{k} a_{i l} b_{l j}
$$

According to formula (3), there are $(\mathrm{m} \times \mathrm{k} \times \mathrm{n})$ times addition and multiplication, and the time complexity of the serial algorithm is $\mathrm{O}\left(\mathrm{n}^{3}\right)$.

\subsection{Parallel computing}

Parallel computing is a form of computation in which many calculations are

carried out simultaneously. Parallelism has been employed in high-performance computing, but it due to the physical constraints.

Analyzing the definition mentioned above, the calculation of each element of the matrix $\mathrm{C}$ is irreverent to each other. Therefore, the addition and multiplication could both be implemented parallel on a multiprocessor platform.

There are some classical algorithms, including DNS algorithm, Cannon algorithm, Fox algorithm, algorithm, Systolic algorithm. All of them are based on the larger size of the matrix in accordance with the actual use of the processor into a number of small matrix. By solving the partial solution of the small matrix multiplication, the partial solution is finally obtained by accumulating the solution of the original matrix.

The performance of a parallel matrix multiplication algorithm can be estimated by running time $t_{p}(n)$ and the number of processors $\mathrm{P}(\mathrm{n})$.

Table 1 shows the performance of the classical algorithms[16].

Table 1: Performance of the classical algorithms

\begin{tabular}{lllll}
\hline Parameters & DNS & Cannon & Fox & systolic \\
\hline $\mathrm{t}_{p}(\mathrm{n})$ & $\log \mathrm{n}$ & $\mathrm{n}$ & $\mathrm{nlogn}$ & $\mathrm{n}$ \\
$\mathrm{P}(\mathrm{n})$ & $\mathrm{n}^{3}$ & $\mathrm{n}^{2}$ & $\mathrm{n}^{2}$ & $\mathrm{n}^{2}$ \\
\hline
\end{tabular}




\section{3. $\Pi_{\mathrm{MM}}$ for Matrix Multiplication}

115

In the system, the initial sequenced membranes denote the input and output membranes, and the generated zero membrane as a processor computes a value of product. The details of the system will be given in this section.

\subsection{Definition}

120 membranes can be defined as:

$$
\Pi_{M M}=\left(O, \mu, \Delta, \omega_{1}, \cdots, \omega_{m}, R, i_{o}\right)
$$

(1) $\mathrm{O}$ is a finite and non-empty alphabet of objects, $\mathrm{O}=\left\{\mu, \theta, \xi, \Psi_{1}, \Psi_{2}, \Psi_{3}, \Psi_{4}, \Psi_{5}\right\}$ $\cup\left\{\mu_{j}, \beta_{j}, 1 \leq j \leq m\right\} \cup\left\{a_{i 1}, a_{i 2}, b_{i 1}, b_{i 2}, d_{i 1}, d_{i 2}, \gamma_{i}, 1 \leq i \leq k\right\} \cup\left\{c_{i 1}, c_{i 2}, 0 \leq\right.$ $i \leq k\} \cup\left\{+{ }_{i},-{ }_{i},+^{i},-{ }^{i}, 1 \leq i \leq k\right\}$

(2) $\mu$ is the membrane structure composed of m membranes, each region labeled as $1,2, \ldots, \mathrm{m}$, the number $\mathrm{m}$ is decided by the row of matrix A, shown in Fig.1. Each membrane has the fraction of scalar for a row.

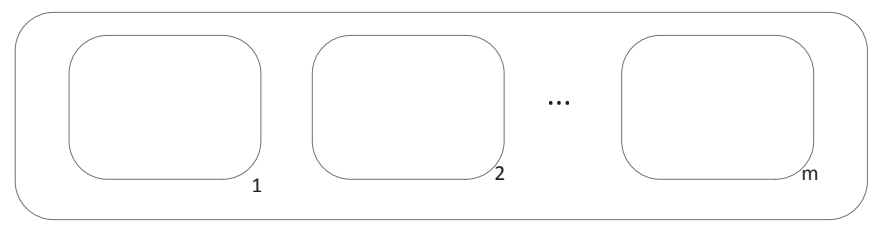

Figure 1: Membrane structure

(3) $\Delta$ is an embedded $\mathrm{P}$ system for completing fraction arithmetic operation and fraction reduction that we have been constructed in Ref. [9] and Ref. [10] Assuming this system is existed in every membrane when it generated.

(4) $\omega_{i}$ is multi-sets, representing the objects for $i_{t h}$ row of matrix initially present in the regions $i$. In initial state, $\omega_{i}=\Phi$. 
(5) The rules in $\mathrm{R}$ should have a priority, and the explanations of some rules are given here, $k$ indicates the priority: When $\mathrm{k}=1$, the corresponding rule will have the highest priority.

All of the rules that can be applied must be applied simultaneously. The marked output membranes are never dissolved.

\subsection{Rule classification}

160

All rules of the system can be classified into three categories composed of eight rule sets by different catalyst, because synchronization is guaranteed by 
arranging some special objects in these rules.

$$
\mathrm{R}=\left\{R_{1}, R_{2}, R_{3}, R_{4}, R_{5}, R_{6}, R_{7}, R_{8}\right\}
$$

The rule set $R_{1}$ which control the whole computing procedure for guarantying synchronization is introduced here. The rule sets $R_{2}$ and $R_{3}$ are used to

Next, input matrix B by columns, insert label $\Psi_{1}$ after a column. The rule set $R_{3}$ is for imputing matrix $\mathrm{B}$. 


$$
R_{3}=\left\{r_{1}:\left(\left.b_{i 1} \rightarrow\left(b_{i 1}, i n\right)\right|_{\xi}, 1 \leq i \leq k, 1\right)\right\} \cup
$$

$$
\begin{aligned}
& \left\{r_{2}:\left(\left.b_{i 2} \rightarrow\left(b_{i 2}, i n\right)\right|_{\xi}, 1 \leq i \leq k, 1\right)\right\} \cup \\
& \left\{r_{3}:\left(+\left.{ }_{i} \rightarrow\left(+{ }_{i}, i n\right)\right|_{\xi}, 1 \leq i \leq k, 1\right)\right\} \cup \\
& \left\{r_{4}:\left(-\left.{ }_{i} \rightarrow\left(-{ }_{i}, i n\right)\right|_{\xi}, 1 \leq i \leq k, 1\right)\right\}
\end{aligned}
$$

\subsection{Membrane division and permeation}

Divide the generated membranes to obtain the copies of matrix B by $\Psi_{3}$. membrane.

$$
\begin{aligned}
R_{4}= & \left\{r_{1}:\left(\Psi_{3}\left[{ }_{0}\right]_{0} \rightarrow\left[{ }_{0}\right]_{0}\left[_{0}\right]_{0}, 1\right)\right\} \cup \\
& \left.\left\{r_{2}: \beta_{j}[0]_{0} \rightarrow\left[{ }_{j} \beta\left[{ }_{0}\right]_{0}\right]_{j}, 1 \leq j \leq m, 1\right)\right\}
\end{aligned}
$$
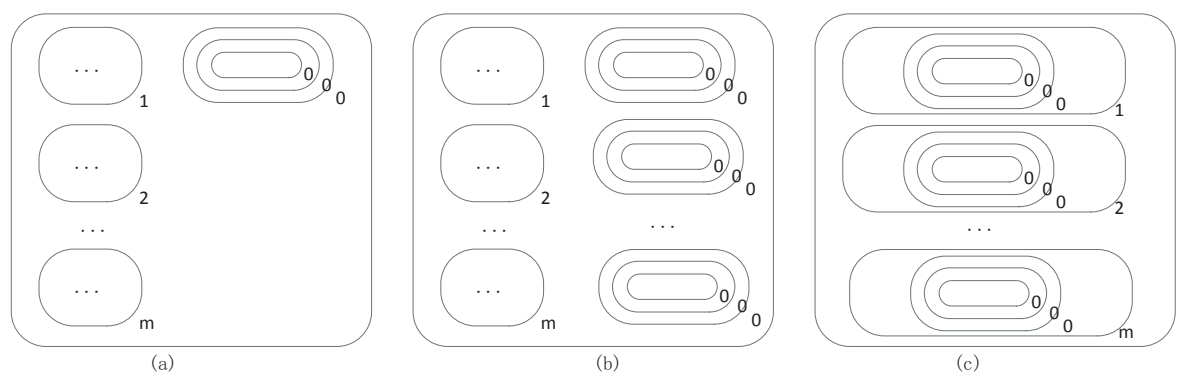

Figure 2: Division and permeation

\subsection{Calculation}

200

205

The rule set $R_{5}$ is used to send objects into inner membranes in each marked membrane.

$$
\begin{aligned}
R_{5}= & \left\{r_{1}:\left(\left.a_{i 1} \rightarrow\left(a_{i 1}, i n\right)\right|_{\beta}, 1 \leq i \leq k, 1\right)\right\} \cup \\
& \left\{r_{2}:\left(\left.a_{i 2} \rightarrow\left(a_{i 2}, i n\right)\right|_{\beta}, 1 \leq i \leq k, 1\right)\right\} \cup \\
& \left\{r_{3}:\left(+\left._{i} \rightarrow\left(+_{i}, i n\right)\right|_{\beta}, 1 \leq i \leq k, 1\right)\right\} \cup \\
& \left\{r_{4}:\left(-\left.{ }_{i} \rightarrow\left(-{ }_{i}, i n\right)\right|_{\beta}, 1 \leq i \leq k, 1\right)\right\} \cup \\
& \left\{r_{5}:\left(\left.a_{i 1} \rightarrow d_{i 1}\left(a_{i 1}, i n\right)\right|_{\Psi 4}, 1 \leq i \leq k, 1\right)\right\} \cup \\
& \left\{r_{6}:\left(\left.a_{i 2} \rightarrow d_{i 2}\left(a_{i 2}, i n\right)\right|_{\Psi 4}, 1 \leq i \leq k, 1\right)\right\} \cup \\
& \left\{r_{7}:\left(+_{i} \rightarrow++\left.^{i}\left(+_{i}, i n\right)\right|_{\Psi_{4}}, 1 \leq i \leq k, 1\right)\right\} \cup \\
& \left\{r_{8}:\left(-{ }_{i} \rightarrow-\left.{ }^{i}\left(-{ }_{i}, i n\right)\right|_{\Psi_{4}}, 1 \leq i \leq k, 1\right)\right\} \cup
\end{aligned}
$$




$$
\begin{aligned}
& \left\{r_{9}:\left(\left.a_{i 1} \rightarrow d_{i 1}\right|_{\Psi_{4}}, 1 \leq i \leq k, 2\right)\right\} \cup \\
& \left\{r_{10}:\left(\left.a_{i 2} \rightarrow d_{i 2}\right|_{\Psi_{4}}, 1 \leq i \leq k, 2\right)\right\} \cup \\
& \left\{r_{11}:\left(+{ }_{i} \rightarrow+\left.{ }^{i}\right|_{\Psi_{4}}, 1 \leq i \leq k, 2\right)\right\} \cup \\
& \left.\left\{r_{12}:\left.\left(-{ }_{i} \rightarrow-{ }^{i}, i n\right)\right|_{\Psi_{4}}, 1 \leq i \leq k, 2\right)\right\} \cup \\
& \left\{r_{13}:(\beta \rightarrow \beta(\beta, i n), 2), r_{14}:\left(\beta \Psi 2 \rightarrow \Psi_{4}, 1\right), r_{15}: \beta \rightarrow \lambda, 3\right\}
\end{aligned}
$$

The rule set $R_{6}$ is used to realize formula $\frac{c_{i 1}}{c_{i 2}}=\frac{b_{i 1}}{b_{i 2}} \times \frac{d_{i 1}}{d_{i 2}}$ for multiplication.

$$
\begin{aligned}
& R_{6}=\left\{r_{1}:\left(\left.b_{i 1} \rightarrow\left(\xi_{1} t_{1}, i n_{\Delta}\right)\right|_{\Psi 4}, 1 \leq i \leq k, 3\right)\right\} \cup \\
&\left\{r_{2}:\left(\left.b_{i 2} \rightarrow\left(\xi_{1} t_{2}, i n_{\Delta}\right)\right|_{\Psi 4}, 1 \leq i \leq k, 3\right)\right\} \cup \\
&\left\{r_{3}:\left(\left.d_{i 1} \rightarrow\left(\xi_{2} t_{1}, i n_{\Delta}\right)\right|_{\Psi 4}, 1 \leq i \leq k, 3\right)\right\} \cup \\
&\left\{r_{4}:\left(\left.d_{i 2} \rightarrow\left(\xi_{2} t_{2}, i n_{\Delta}\right)\right|_{\Psi 4}, 1 \leq i \leq k, 3\right)\right\} \cup \\
&\left\{r_{5}:\left(+\left._{i} \rightarrow \gamma_{i}\left(+\times, i n_{\Delta}\right)\right|_{\Psi 4}, 1 \leq i \leq k, 3\right)\right\} \cup \\
&\left\{r_{6}:\left(\left.{ }^{i} \rightarrow \gamma_{i}\left(-\times, i n_{\Delta}\right)\right|_{\Psi 4}, 1 \leq i \leq k, 3\right)\right\} \cup \\
&\left\{r_{7}:\left(+\left.^{i} \rightarrow \gamma_{i}\left(+\times, i n_{\Delta}\right)\right|_{\Psi 4}, 1 \leq i \leq k, 3\right)\right\} \cup \\
&\left\{r_{8}:\left(-\left.^{i} \rightarrow \gamma_{i}\left(-\times, i n_{\Delta}\right)\right|_{\Psi 4}, 1 \leq i \leq k, 3\right)\right\} \cup \\
&\left\{r_{9}:\left(\left.t_{1} \rightarrow c_{i 1}\right|_{\gamma_{i}}, 1 \leq i \leq k, 1\right)\right\} \cup \\
&\left\{r_{10}:\left(\left.t_{2} \rightarrow c_{i 2}\right|_{\gamma_{i}}, 1 \leq i \leq k, 1\right)\right\} \cup \\
&\left\{r_{11}:\left(+\gamma_{i} \rightarrow+{ }_{i}, 1 \leq i \leq k, 1\right)\right\} \cup \\
&\left\{r_{12}:\left(-\gamma_{i} \rightarrow-{ }_{i}, 1 \leq i \leq k, 1\right)\right\}
\end{aligned}
$$

After multiplication, the rule set $R_{7}$ is used to sum up.

$$
\begin{aligned}
R_{7}= & \left\{r_{1}:\left(\left.c_{i 1} \rightarrow\left(\xi_{1} t_{1}, i n_{\Delta}\right)\right|_{\Psi 4}, 0 \leq i \leq 1,4\right)\right\} \cup \\
& \left\{r_{2}:\left(\left.c_{i 2} \rightarrow\left(\xi_{1} t_{2}, i n_{\Delta}\right)\right|_{\Psi 4}, 0 \leq i \leq 1,4\right)\right\} \cup \\
& \left\{r_{3}:\left(\left.c_{i 1} \rightarrow\left(\xi_{1} t_{1}, i n_{\Delta}\right)\right|_{\Psi 4}, 2 \leq i \leq k, 4\right)\right\} \cup \\
& \left\{r_{4}:\left(\left.c_{i 2} \rightarrow\left(\xi_{1} t_{2}, i n_{\Delta}\right)\right|_{\Psi 4}, 2 \leq i \leq k, 4\right)\right\} \cup \\
& \left\{r_{5}:\left(+\left._{i} \rightarrow \gamma\left(++, i n_{\Delta}\right)\right|_{\Psi 4}, 0 \leq i \leq 1,4\right)\right\} \cup \\
& \left\{r_{6}:\left(-\left._{i} \rightarrow \gamma\left(-+, i n_{\Delta}\right)\right|_{\Psi 4}, 0 \leq i \leq 1,4\right)\right\} \cup \\
& \left\{r_{7}:\left(+\left._{i} \rightarrow\left(++, i n_{\Delta}\right)\right|_{\Psi 4}, 2 \leq i \leq k, 4\right)\right\} \cup \\
& \left\{r_{8}:\left(\left.{ }_{i} \rightarrow\left(-+, i n_{\Delta}\right)\right|_{\Psi 4}, 2 \leq i \leq k, 4\right)\right\} \cup \\
& \left\{r_{9}:\left(\left.t_{1} \rightarrow c_{01}\right|_{\gamma}, 1\right), r_{10}:\left(\left.t_{2} \rightarrow c_{02}\right|_{\gamma}, 1\right)\right\} \cup \\
& \left\{r_{11}:\left(+\rightarrow \mu+\left.{ }_{0}\right|_{\gamma} 1\right), r_{12}:\left(-\rightarrow \mu-\left.{ }_{0}\right|_{\gamma} 1\right)\right\} \cup \\
& \left\{r_{13}:\left(\mu^{k} \rightarrow \Psi 5,1\right), r_{14}:\left(\gamma^{k-1} \rightarrow \mu, 1\right)\right\}
\end{aligned}
$$

The rule set $R_{8}$ is used to dissolve the inner membrane and output the 
solution in the marked membrane.

$$
\begin{aligned}
R_{8}= & \left\{r_{1}:\left(\left.c_{i 1} \rightarrow\left(c_{(i+1) 1}, \text { out }\right)\right|_{\Psi 5}, 0 \leq i<k, 2\right)\right\} \cup \\
& \left\{r_{2}:\left(\left.c_{i 2} \rightarrow\left(c_{(i+1) 2}, \text { out }\right)\right|_{\Psi 5}, 0 \leq i<k, 2\right)\right\} \cup \\
& \left\{r_{3}:\left(+\left._{i} \rightarrow\left(+_{(i+1)}, \text { out }\right)\right|_{\Psi 5}, 0 \leq i<k, 2\right)\right\} \cup \\
& \left\{r_{4}:\left(\left.{ }_{i} \rightarrow\left({ }_{(i+1)}, \text { out }\right)\right|_{\Psi 5}, 0 \leq i<k, 2\right)\right\} \cup \\
& \left\{r_{5}:(\Psi 5 \rightarrow \delta, 3), r_{6}:([\delta] \rightarrow \lambda, 3)\right\}
\end{aligned}
$$

\subsection{Complexity analysis}

In this part, an analysis of $\Pi_{\mathrm{MM}}$ is given. Suppose applying one rule will cost one time slice denoted as $t_{\Omega}$, so some rules are using concurrently will also cost one time slice. Besides, the process which includes input, computing and output by calculating component $\Delta$ will cost another one time slice denoted as $t_{\Delta}$.

As mentioned above, the system has $\left(\mathrm{n}^{*} \mathrm{~m}+\mathrm{m}\right)$ membrane for $\mathrm{n}$ unknowns, each has one row of equations, and $n$ calculating component, so the arithmetic operation are parallel execution.

Assuming $t_{\Omega}$ is less than $t_{\Delta}$, can be ignored, therefore the complexity of the system can be worked out. So the sum of operation capacity is $(\mathrm{mk}+\mathrm{nk}+3 \mathrm{k})$.

Compare the complexity of $\Pi_{\mathrm{MM}}$ to that of serial computing and parallel computing. The complexity of serial computing method is $\mathrm{O}\left(n^{3}\right)$. The complexity of parallel computing method is $\mathrm{O}\left(n^{3} / p\right)$, the value of $\mathrm{p}$ is an integer among 1 to $n$. The complexity of $\Pi_{\mathrm{MM}}$ is $\mathrm{O}\left(n^{2}\right)$.

Obviously, only when $\mathrm{p}=\mathrm{n}$, the complexity of parallel computing method is $\mathrm{O}\left(n^{2}\right)$, which equivalent to complexity of $\Pi_{\mathrm{MM}}$. So $\Pi_{\mathrm{MM}}$ is the best to solve the problem.

\section{An Example For $\Pi_{M M}$}

For describing how the $\Pi_{\mathrm{MM}}$ working, here we give an example to illustrate the whole solving procedure. 
Given the matrix A $\left(4^{*} 5\right)$ matrix $\mathrm{B}\left(5^{*} 3\right)$ are as follows:

$$
A \times B=\left[\begin{array}{ccccc}
3 & -2 & -1 & 2 & 1 \\
2 & 4 & \frac{3}{2} & -3 & -\frac{3}{2} \\
1 & -3 & 2 & 1 & 2 \\
-1 & 2 & -\frac{5}{2} & 4 & 1
\end{array}\right]\left[\begin{array}{ccc}
-2 & 1 & \frac{3}{2} \\
4 & \frac{5}{2} & -1 \\
2 & 3 & 5 \\
-1 & 3 & 2 \\
3 & 1 & 2
\end{array}\right]
$$

In the preprocessing, the values of matrix are converted into objects in membrane system. Each number is denotes as three kinds of object: positive or negative symbol; The absolute value of the number is in faction, two objects $a_{i 1}$ for numerator, one object $a_{i 2}$ for denominator. So the matrix A are transformed to the objects set by rows shown in Table 2 . On the other side, the matrix B is transformed to the objects set by columns in Table 3 .

Table 2: The objects of matrix A

\begin{tabular}{llllll}
\hline Row $/$ Col & $1^{\text {st }} \mathrm{col}$ & $2^{\text {nd }} \mathrm{col}$ & $3^{\text {rd }} \mathrm{col}$ & $4^{\text {th }} \mathrm{col}$ & $5^{\text {th }} \mathrm{col}$ \\
\hline $1^{\text {st }}$ row & $a_{11}^{3}, a_{12},+_{1}$ & $a_{21}^{2}, a_{22},--_{2}$ & $a_{31}, a_{32},-3$ & $a_{41}^{2}, a_{42},+_{4}$ & $a_{51}, a_{52},+_{5}$ \\
$2^{\text {nd }}$ row & $a_{11}^{2}, a_{12},+_{1}$ & $a_{21}^{4}, a_{22},+_{2}$ & $a_{31}^{3}, a_{32}^{2},+_{3}$ & $a_{41}^{3}, a_{42},-{ }_{4}$ & $a_{51}^{3}, a_{52}^{2},-_{5}$ \\
$3^{\text {rd }}$ row & $a_{11}, a_{12},+_{1}$ & $a_{21}^{3}, a_{22},-2$ & $a_{31}^{2}, a_{32},+_{3}$ & $a_{41}, a_{42},+_{4}$ & $a_{51}^{2}, a_{52},+_{5}$ \\
$4^{\text {th }}$ row & $a_{11}, a_{12},-1$ & $a_{21}^{2}, a_{22},+_{2}$ & $a_{31}^{5}, a_{32}^{2},-3$ & $a_{41}^{4}, a_{42},+_{4}$ & $a_{51}, a_{52},+_{5}$ \\
\hline
\end{tabular}

Table 3: The objects of matrix B

\begin{tabular}{llllll}
\hline Col/Row & $1^{\text {st }}$ row & $2^{\text {nd }}$ row & $3^{\text {rd }}$ row & $4^{\text {th }}$ row & $5^{\text {th }}$ row \\
\hline $1^{\text {st }}$ col & $b_{11}^{2}, b_{12},-_{1}$ & $b_{21}^{4}, b_{22},+_{2}$ & $b_{31}^{2}, b_{32},+_{3}$ & $b_{41}, b_{42},-_{4}$ & $b_{51}^{2}, b_{52},+_{5}$ \\
$2^{\text {nd }}$ col & $b_{11}, b_{12},+_{1}$ & $b_{21}^{5}, b_{22}^{2},+_{2}$ & $b_{31}^{3}, b_{32},+_{3}$ & $b_{41}^{3}, b_{42},+_{4}$ & $b_{51}, b_{52},+_{5}$ \\
$3^{r d}$ col & $b_{11}^{3}, b_{12}^{2},+_{1}$ & $b_{21}, b_{22},+_{2}$ & $b_{31}^{5}, b_{32},+_{3}$ & $b_{41}^{2}, b_{42},+_{4}$ & $b_{51}^{2}, b_{52},+_{5}$ \\
\hline
\end{tabular}

After the transformation, the objects of matrix A input into initial membranes shown in Table 2 . In this example, $\mathrm{k}=5$, input $\mu^{5}$ and $\mu_{1}$ as catalyst. 
Apply the rules $\left(r_{1}, r_{2}\right)$ in $R_{1}$ and $\left(r_{1}, r_{2}, r_{3}, r_{4}\right)$ in $R_{2}$ to send objects into membrane, Fig. 3 shows the inputting procedure.

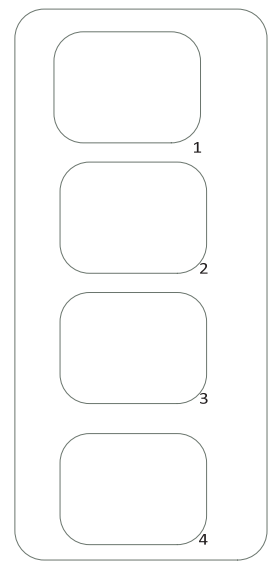

(a)

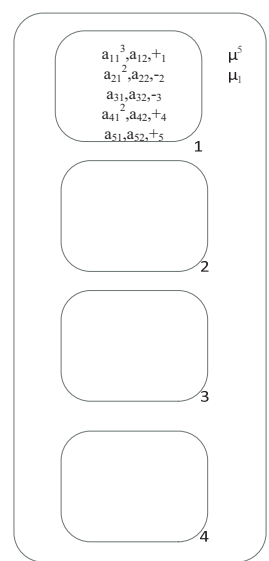

(b)

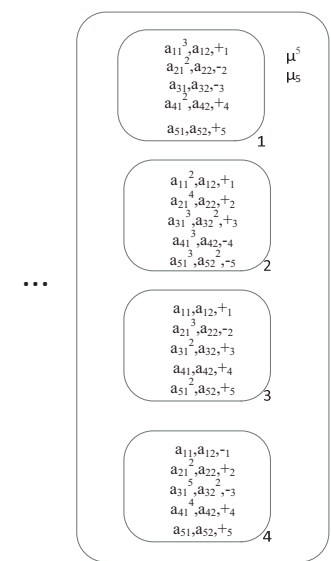

(c)

Figure 3: Inputting procedure

Then generate the new membranes for matrix B. Assuming each membrane operations.

Input $\theta$ by 3 times to generate new membranes by rules $\left(r_{3}, r_{4}\right)$ in $R_{1}$ shown in Fig. 4.

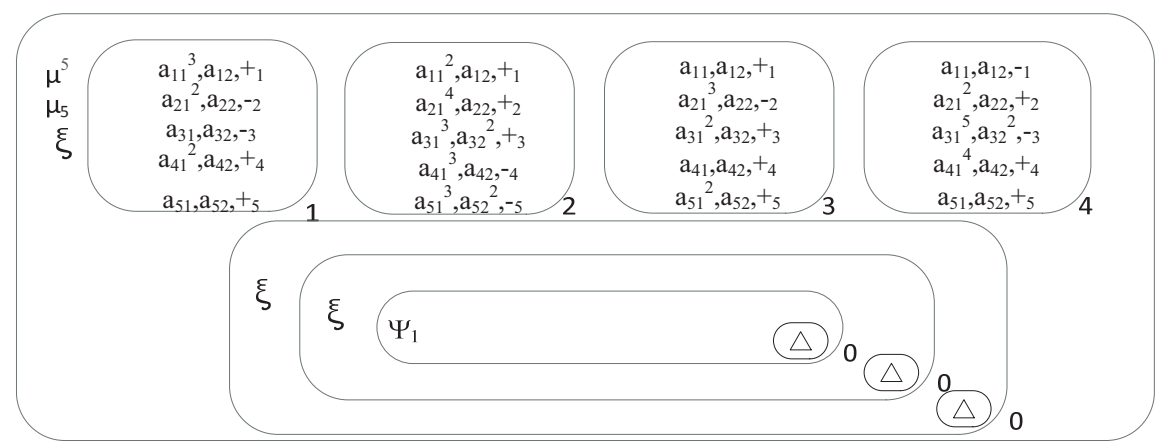

Figure 4: Generating membranes

Input matrix B into embedded membranes by columns according to rules ( 
$\left.r_{1}, r_{2}, r_{3}, r_{4}\right)$ in $R_{3}$ shown in Fig. 5.

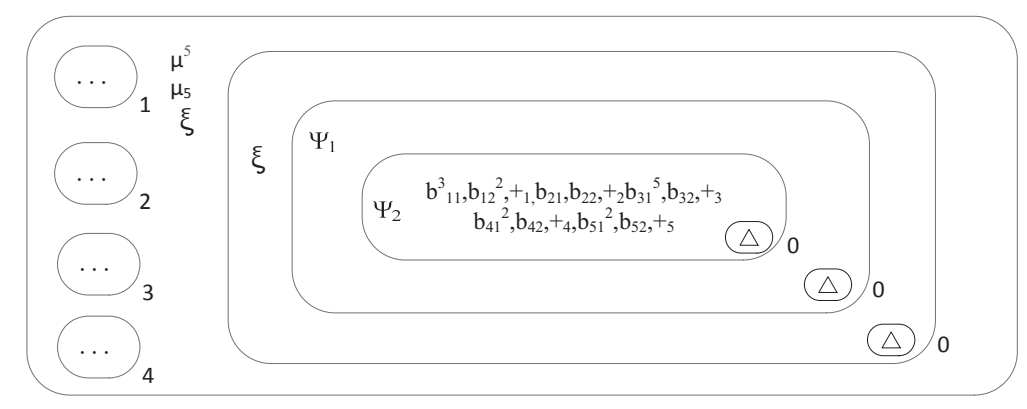

Figure 5: Input first column of matrix B

Repeat the process until all columns are in the membranes shown in Fig. 6.

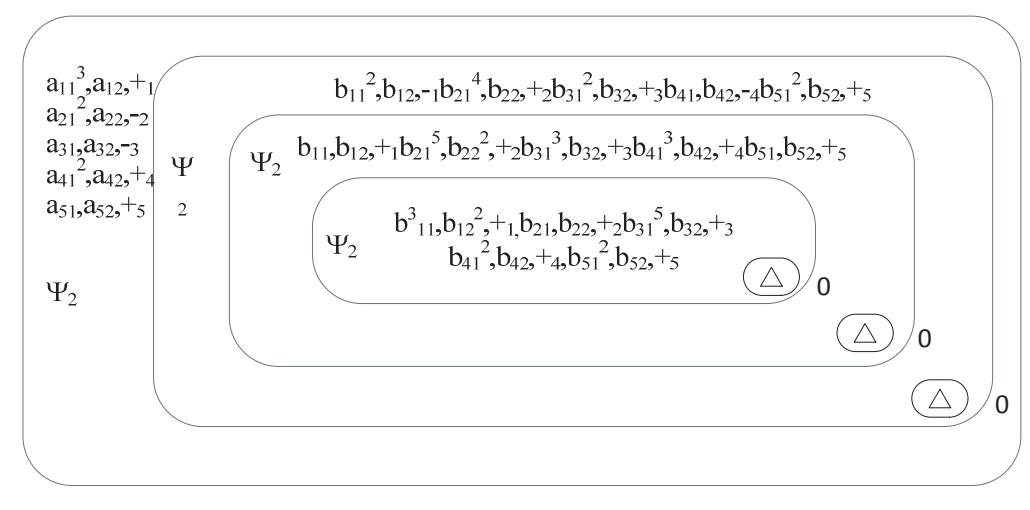

Figure 6: End of inputting

Input catalyst $\Psi_{3}$ two times to applying rules $r_{1}$ in $r_{4}$ to get the membrane structure in Fig. 7 , then input catalyst $\beta_{j}(1 \leq j \leq m)$ to applying rules $r_{2}$ in $R_{4}$ to get the membrane structure in Fig. 8 . 


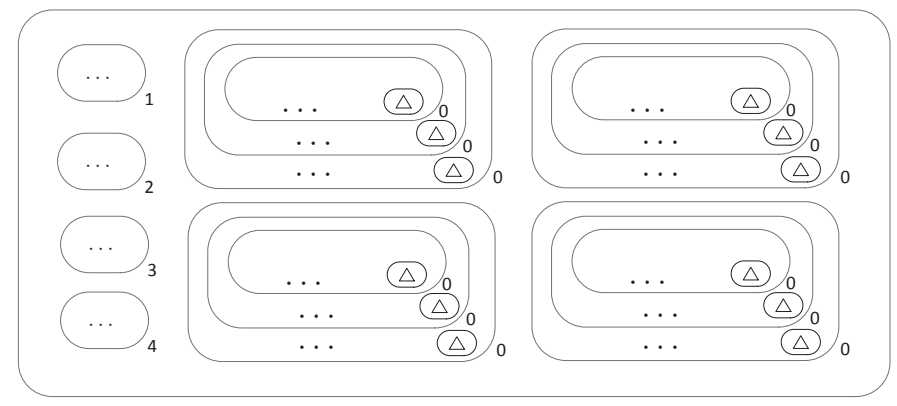

Figure 7: Membrane division

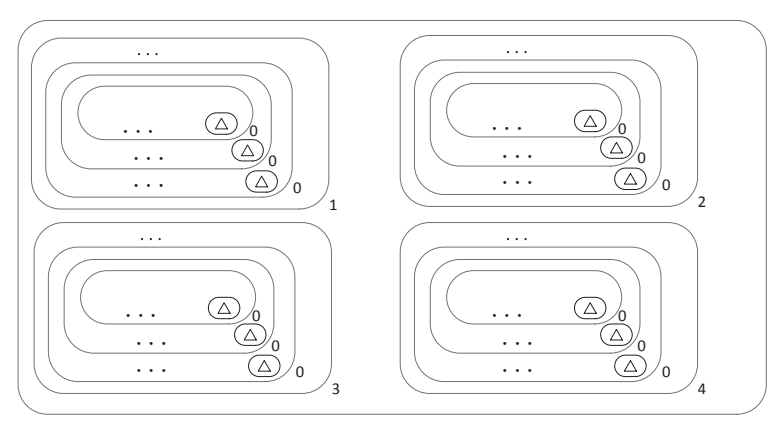

Figure 8: Membrane permeation

290 calculation by rules in $\left(r_{1}\right.$ to $\left.r_{1} 5\right)$ in $R_{5}$ shown in Fig.9.

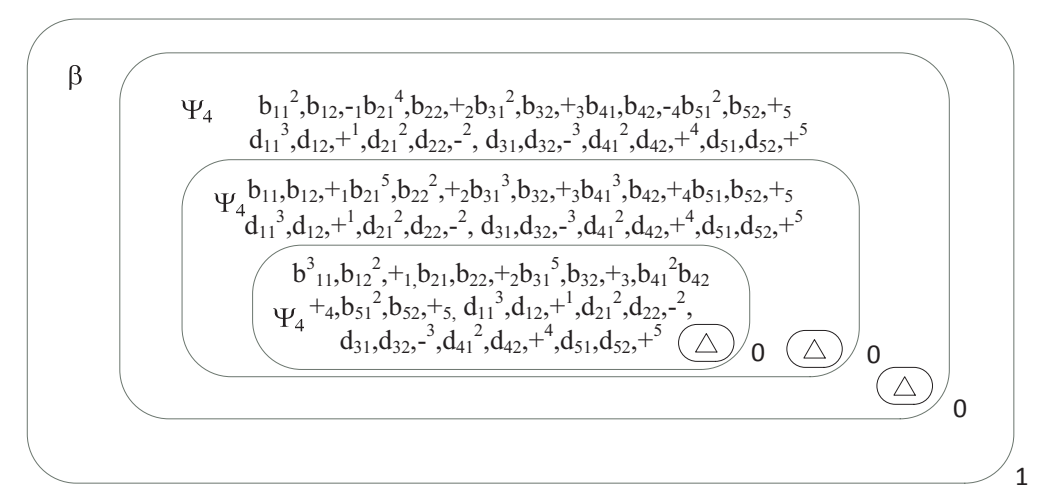

Figure 9: Preparing calculation 
Apply the rule set $R_{6}$ is used to complete the multiplication shown in Fig. 10 and the rule set $R_{7}$ is used to complete the addition shown in Fig. 11.

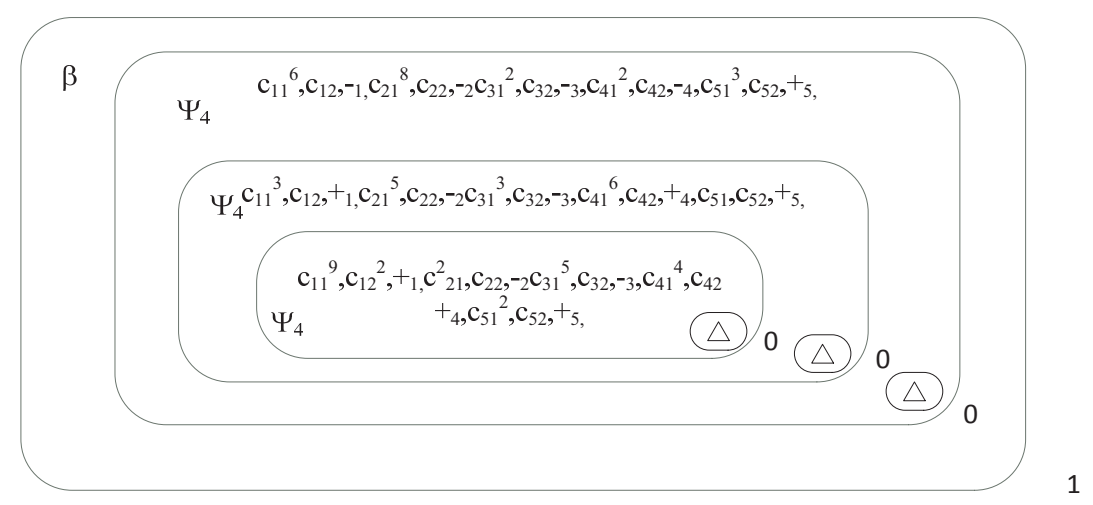

Figure 10: Multiplication calculation

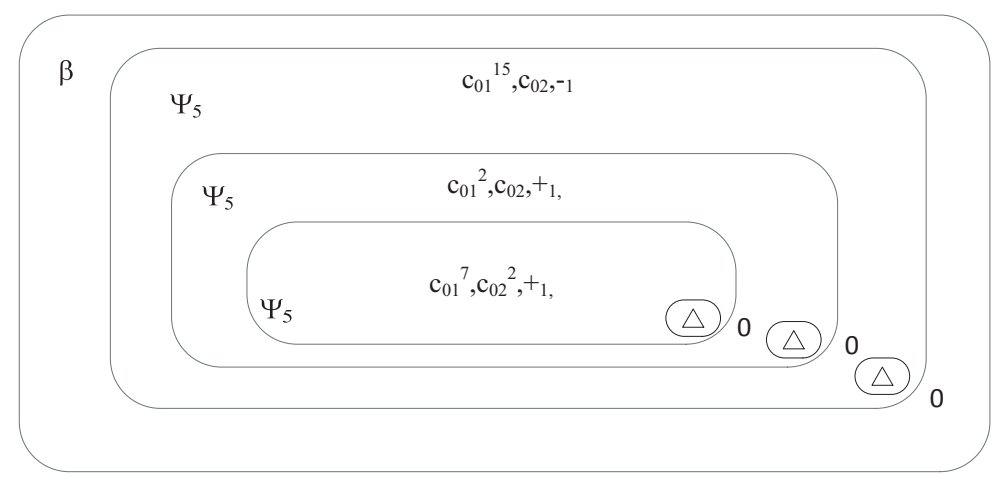

Figure 11: Addition calculation

After all the calculation rules have been applied, the results are in each 295 marked membrane shown in Fig. 12. The solution of the product can be read out from the membrane. 


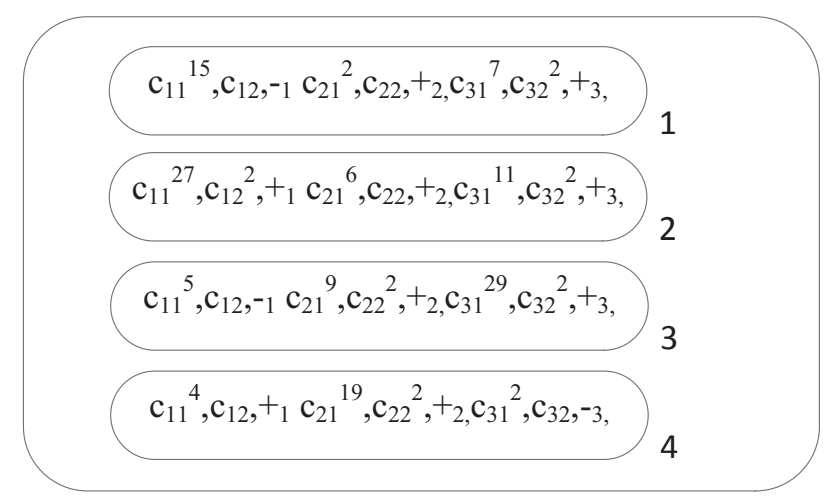

Figure 12: Results of matrix multiplication

\section{Conclusions}

Matrix multiplication is one of important matrix arithmetic operations. In this paper, we proposed a membrane system to complete matrix multiplication

\section{References}

[1] G. Păun, Computing with membranes, Journal of Computer and System Sciences 61 (1) (2000) 108-143. doi:10.1006/jcss.1999.1693. 
[2] A. Arkin, J. Ross, Computational functions in biochemical reaction networks., Biophysical Journal 67 (2) (1994) 560-578. doi:10.1016/S0006-3495(94)80516-8.

[3] A. Obtulowicz, P system for solving sat problem, Journal of Information Science and Technology 4 (1-2) (2001) 195-202.

[4] M. Madhu, K. Krithivasan, P systems with membrane creation: Universality and efficiency, Third International Conference, MCU 2055 (2001) 276-287. doi:10.1007/3-540-45132-3_19.

[5] J. Xiao, Y. Huang, Z. Cheng, J. He, Y. Niu, A hybrid membrane evolutionary algorithm for solving constrained optimization problems, Optik International Journal for Light and Electron Optics 125 (2) (2014) 897-902. doi:10.1016/j.ijleo.2013.08.032.

325 [6] P. Guo, H. Zhang, Arithmetic operation in single membrane, Proc. of International Conference on Computer Science and Software Engineering 3 (2008) 532-535. doi:10.1109/CSSE. 2008.1212.

[7] P. Guo, H. Chen, H. Zheng, Arithmetic expression evaluations with membranes, Chinese Journal of Electronics 23 (1) (2014) 55-60.

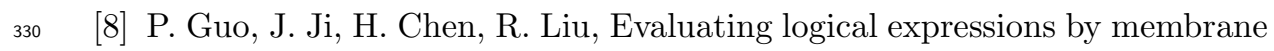
systems, Chinese Journal of Electronics 23 (2) (2014) 278-283.

[9] P. Guo, H. Zhang, H. Chen, J. Chen, Fraction arithmetic operations performed by p systems, Chinese Journal of Electronics 22 (4) (2013) 689-694.

[10] P. Guo, H. Zhang, H. Chen, Fraction reduction in membrane systems, Scientific World Journal 2014 (2014) 1-10. doi:10.1155/2014/858527.

[11] A. Vitale, G. Mauri, C. Zandron, Simulation of a bounded symport /antiport p system with brane calculi, BioSystems 91 (3) (2008) 558-571. doi: $10.1016 /$ j.biosystems . 2007.01.008. 
[12] J. He, J. Xiao, X. Liu, T. Wu, T. Song, A novel membrane-inspired algorithm for optimizing solid waste transportation, Optik - International Journal for Light and Electron Optics 126 (23) (2015) 3883-3888. doi:10.1016/j.ijleo.2015.07.152.

[13] C. Mart, G. Păun, J. Pazos, Tissue p systems, Theoretical Computer Science 296 (2) (2003) 295-326. doi:10.1016/S0304-3975(02)00659-X.

[14] M. Ionescu, G. Păun, T. Yokomori, Spiking neural p systems, FundamentaInformaticae 71 (2-3) (2006) 279-308.

[15] C. Liu, D. Chen, F. Wan, Multiobjective learning algorithm based on membrane systems for optimizing the parameters of extreme learning machine, Optik - International Journal for Light and Electron Optics 127 (4) (2016) 1909-1917. doi:10.1016/j.ijleo.2015.11.140.

[16] P. Chen, X. Fan, Several kinds of parallel algorithm for matrix multiplication comparative analysis, Journal of Xinjiang Normal University(Natural Sciences Edition) 31 (3) (2012) 5-10. 